\title{
Exercise: from physiology to bedside to physiology
}

\author{
Jin $\operatorname{Han}^{1} \cdot$ Darrell Neufer $^{2} \cdot$ Henriette Pilegaard $^{3}$ \\ Published online: 6 February 2020 \\ (C) Springer-Verlag GmbH Germany, part of Springer Nature 2020
}

Evidence demonstrating the benefits of physical activity have rapidly increased over recent years based on research showing how exercise improves function and structure at the molecular and cellular levels, clearly conveying a physiological role in vitro and in vivo. The changes in the functional properties of cells and organs may be associated with protection against the pathophysiology of various diseases. Understanding the nature of physiology in relation to physical activity helps develop new interventions and therapeutic strategies to treat disease. Conversely, clinical knowledge can also inspire a breadth of physiological research.

This issue of Pflügers Archiv - European Journal of Physiology provides a series of review articles and original papers focusing on the translational promise of exercise through physiology. Covered in this issue is the role of exercise as a regulator of mitochondrial homeostasis through posttranslational modifications, which in turn affect processes such as mitochondrial biogenesis and mitophagy, and which can also serve as the basis for developing innovative pharmaceutical strategies to mimic exercise therapy. Exercise can also serve as an inducer of cardiac structure and functional adaptation which is vital in developing exercise therapies in relation to heart disease. The role of Epac in regulating the mitochondrial biogenesis regulator PGC- $1 \alpha$ and fatty acid metabolism in the skeletal muscle is also covered. Also presented are novel insights regarding the capability of exercise as a regulator of the immune system, which may be antigenspecific or cytokine-dependent based on exercise modalities, as an influencer of circadian rhythm based on proteins regulated by exercise during day and night, or as an effector of glucose response. We thank all for our colleagues' contribution to this special issue. Again, we appreciate all the support to publish this from Armin Kurtz and Yung E Earm.

Jin Han

phyhanj@inje.ac.kr

Darrell Neufer

neuferp@ecu.edu

Henriette Pilegaard

hpilegaard@bio.ku.dk

1 Department of Physiology, College of Medicine, Cardiovascular and Metabolic Disease Center, Inje University, 75 Bokji-ro, Busanjin-gu, Busan 614-735, South Korea

2 East Carolina Diabetes and Obesity Institute, Departments of Physiology and Kinesiology, East Carolina University, 115 Heart Drive, ECHI - Mail Stop 743, Greenville, NC 27834, USA

3 Section for Cell Biology and Physiology, Department of Biology, University of Copenhagen, Universitetsparken 13, 2100 Copenhagen $\varnothing$, Denmark 